\title{
FM6: Summary of Session \#5 on Accretion and Feedback in Active Galactic Nuclei
}

\author{
Laura Brenneman \\ Smithsonian Astrophysical Observatory \\ MS-4, 60 Garden St., Cambridge, MA 02138 USA \\ email: lbrenneman@cfa.harvard.edu
}

\begin{abstract}
Focus Meeting 6 of the IAU 2015 Symposium centered around the topic of "X-ray Surveys of the Hot and Energetic Universe." Within this two-day meeting seven sessions (31 total talks) were presented, whose topics included galaxy cluster physics and evolution, cluster cosmological studies, AGN demographics and X-ray binary populations, first quasars, accretion and feedback, large-scale structures, and normal and starburst galaxies. Herein, I summarize the results presented during session \#5, which focused on AGN accretion and feedback. Six authors contributed their work to our session: Laura Brenneman, Kazushi Iwasawa, Massimo Gaspari, Michaela Hirschmann, Franz Bauer and Yuan Liu. I provide a brief introduction below, followed by the details of the presentations of each author in the order in which the presentations were given.
\end{abstract}

Keywords. accretion, accretion disks; black hole physics; galaxies: active; galaxies: jets; X-rays: galaxies; infrared: galaxies.

\section{Introduction}

A key outcome of X-ray surveys of active galactic nuclei (AGN) over the past three decades has been an appreciation of the great variety of observed physical properties of AGN and their hosts. While our ability to categorize and classify many thousands of sources to date speaks to the triumph of improving imaging and spectroscopic instruments, techniques and software algorithms, we continually find outliers to observed trends that challenge our present understanding of how galaxies and their supermassive black holes (SMBHs) form and evolve (e.g., > $10^{10} \mathrm{M}_{\odot}$ SMBHs; Wu et al. 2015). Increasingly sophisticated numerical simulations are becoming ever more accurate in their ability to derive the physical conditions in present-day galaxies from initial seeds observed at high redshift, and their results offer us intriguing predictions of what future observatories might find with new capabilities that allow surveys to push down to lower flux limits, both locally and in the distant universe (e.g., Athena, X-ray Surveyor; Nandra et al. 2013, Weisskopf et al. 2015).

Examining the roles of accretion and feedback in shaping the evolution of SMBHs in AGN and their host galaxies over cosmic time is a rapidly growing offshoot of this field of astrophysical research, both theoretically and observationally. In this summary of session \#5 in Focus Meeting 6 (FM6) of the 2015 International Astronomical Union (IAU) Symposium, six authors have contributed their perspectives on recent developments in how accretion and feedback shape their galactic environs. Their topics covered a range of work, including measuring black hole spins with X-ray spectra of AGN, highly ionized gas atmospheres in AGN, the interconnectedness of accretion and feedback processes, the role of SMBH mergers in AGN/host interaction and nuclear activity, extended $\mathrm{Fe} \mathrm{K}$ emission regions in nearby AGN and the increasing evidence for clumpy, cold absorbers in AGN systems. The contributions from each author are summarized in each of the following sections. 


\section{The Role of Black Hole Spin in Shaping Accretion, Feedback and Galaxy Evolution over Cosmic Time}

\section{Brenneman}

A central irony of AGN studies is that SMBHs are the ultimate gravitational sinks in their host galaxies, yet they also fuel the most powerful engines in the universe, influencing star formation, galaxy formation and evolution, and even the evolution of whole clusters of galaxies. The particulars of how SMBHs at the cores of AGN feed off of accretion, and how this accretion ultimately powers the AGN's prodigious energy output remain a mystery, however.

Simply put, how does the energy given off by an AGN so dramatically influence its galactic and intergalactic environment at radii orders of magnitude beyond the SMBH's gravitational sphere of influence? The intimate relationships between a black hole's mass and the surrounding spheroid's mass and stellar velocity dispersion are well documented across many orders of magnitude in black hole mass (e.g., McConnell \& Ma 2013). Similarly, studies of the spatial number density of galaxies vs. their K-band brightness (a proxy for stellar mass) indicate that the number density lies below the value predicted by the cooling cold dark matter halo theory for a given stellar mass at both the high and low ends of the distribution (Bower et al. 2006). The likely explanation for these differences is feedback from stellar winds on the low-mass end and feedback from AGN on the high-mass end, with the theory being that such feedback (radiative and mechanical) heats the interstellar and intergalactic mediums (ISM and IGM), countering the natural tendency of the gas to cool and form stars, and thus regulating the number of stars that can be produced.

Many open issues remain in our exploration of how accretion and feedback are tied together, however. One fundamental mechanism which could relate them is the spin of the SMBH; as one of only two properties that defines an astrophysical black hole, spin dictates the shape of the spacetime within the innermost few tens of radii, thus influencing the innermost accretion flow as well. Further, spin likely plays a critical role in the production of jets (e.g., Blandford \& Znajek 1977). The role (if any) of spin and the nature of the accretion flow in producing AGN winds is as yet unknown, but studies on microquasars have demonstrated that an energetic balance is maintained between the jet and wind outflows that is tied directly to the accretion mode of the central black hole (Neilsen \& Lee 2009). Establishing evidence of a similar trend in AGN is challenging, though, as AGN evolve over much longer timescales and are therefore viewed in snapshots only compared with the much more rapid state transitions shown by microquasars. We must study a distribution of AGN in a variety of spectral states and measure the SMBH spin of each in order to hunt for a similar connection.

The spin of the SMBH is derived from properly modeling the spectrum of the emission reprocessed by the innermost accretion disk closest to the black hole (Reynolds \& Nowak 2003, Brenneman \& Reynolds 2006). Thorough reviews of this so-called "reflection method" are given in Brenneman (2013) and Reynolds (2014), but in brief, thermal (UV) photons from the disk surface radiate outwards, interacting with a relativistic electron plasma in some as yet unknown geometry surrounding the innermost disk known as the "corona." This structure is likely compact and centrally concentrated, as determined by X-ray microlensing observations (Chartas et al.2012, Mosquera et al. 2013). The UV photons are inverse Compton scattered by the electrons, receiving a boost of energy into the X-ray regime. Some fraction of the X-ray photons are then scattered out of the system and into our line of sight, producing the characteristic power-law continuum seen in X-ray spectra of AGN. The rest are scattered back down onto the disk surface where they are reprocessed, exciting a series of fluorescent emission lines from various species, of which 
Fe $\mathrm{K} \alpha$ at $6.4 \mathrm{keV}$ is the most prominent, owing to its abundance in these systems, as well as its high fluorescence yield $(\sim 34 \%)$. A convex continuum from $\sim 7-40 \mathrm{keV}$ is also produced by this process of reflection (the "Compton hump"); peaking at $\sim 20 \mathrm{keV}$, it is shaped by the Fe K edge at low energies and Compton downscattering at high energies.

Spin is derived by modeling the shape of the reflection features in order to determine the radius of the innermost stable circular orbit (ISCO) of the accretion disk. These features are altered by the gravity of the SMBH, becoming more broadened and skewed toward lower energies as the black hole spin is increased in the prograde direction. The ISCO radius depends monotonically on the black hole spin, where we define spin here as a dimensionless quantity $a \equiv c J / G M^{2}$, where $-1 \leqslant a \leqslant 1, J$ is the angular momentum of the hole and $M$ is its mass. Negative values of $a$ represent retrograde configurations between the rotation of the black hole and the innermost disk, positive values represent prograde configurations, and $a=0$ indicates a non-spinning black hole. Several authors have created models to represent the reflection spectrum (Ross \& Fabian 2005, Garcia et al. 2013) and the warping of the spectral features imposed by the black hole (Fabian et al.1989, Laor 1991, Brenneman \& Reynolds 2006, Dauser et al.2010, Dauser et al.2013, Garcia et al. 2014). Within the past decade, the most recent models have allowed the spin of the black hole to be measured as a free parameter in the spectral modeling. The critical assumptions made in these measurements are that (1) the innermost accretion disk extends down to the ISCO, meaning that the derived spin is effectively a lower limit; and (2) no significant reflected emission emanates from within the ISCO (Reynolds \& Fabian 2008).

Measuring spins in SMBHs poses several significant challenges. Firstly, we are currently limited to the inner disk reflection modeling technique in making our measurements, whereas for stellar mass black holes it is also possible to measure their spins via spectral modeling of their thermal continua (McClintock, Narayan \& Steiner 2014), allowing for a cross-check of the measurements between the two methods. Both methods rely on obtaining high signal-to-noise X-ray spectra of these sources; for AGN, this results in long exposure times of only the brightest sources, both making it challenging to obtain the necessary observations and possibly biasing our results toward high spin values (Brenneman et al.2011). Secondly, ionized absorption lines from outflowing gas intrinsic to the AGN along our line of sight to the inner disk are present in over $50 \%$ of AGN (Halpern 1984, Reynolds 1997). This gas, coupled with the often patchy cold absorbing gas of the putative molecular torus in AGN (see $\S 7$ below), can induce spectral curvature that extends up into the Fe $\mathrm{K}$ band, complicating our few of reflection from the innermost disk.

In some cases, applying multiple ionized, partially-covering absorbers to the continuum model results in the same statistical goodness-of-fit as modeling the spectrum with relativistic reflection (Miller, Turner \& Reeves 2008). In order to break these modeling degeneracies and determine the true nature of the spectrum, sensitive, broadband X-ray spectra must be utilized, preferably with multiple observations of the same source seen in several epochs to properly determine the origin of any spectral changes that have taken place. An excellent example of such observations is the XMM-Newton/NuSTAR campaign on the obscured Seyfert-1.9 galaxy NGC 1365 (Risaliti et al.2013, Walton et al.2014). The source was observed four times over a seven month period for $120 \mathrm{ks}$ each. In a given spectrum, the reflection (including absorption) and absorption-only models are statistically indistinguishable below $10 \mathrm{keV}$ with $X M M$ alone, but once the NuSTAR data are added to extend the energy range to $\sim 80 \mathrm{keV}$ (Harrison et al. 2013), the reflection model quite clearly provides a superior fit to the data, even to the naked eye.

Interestingly, NGC 1365 varied dramatically in column density between each observation, ranging from $N_{\mathrm{H}}=10^{22}-10^{24} \mathrm{~cm}^{-2}$. In spite of this variation, when the absorption and continuum were modeled in each case, the remaining signature left behind was nearly 
identical, showing narrow and broad Fe K $\alpha$ lines in emission, Fe XXV and Fe XXVI K $\alpha$ and $\mathrm{K} \beta$ lines in absorption, and a prominent Compton hump. In addition to providing strong visual evidence for the presence of $\sim$ constant reflection features from the innermost disk and outer torus, as well as a highly ionized wind, these features allowed the spin of the $\mathrm{SMBH}$ to be independently measured for each observation. In each case, the measured spin was $a \geqslant 0.97$ to $90 \%$ confidence, lending further support to the presence of relativistic inner disk reflection in NGC 1365, since the spin would not change on such short timescales. Further, adopting the absorption-only model for the source would mean that its intrinsic luminosity due to reprocessed emission from the absorbing material would have to be much higher than for the reflection scenario. An inspection of the infrared data for NGC 1365 shows that this is not the case (Risaliti et al.2013).

NGC 1365 is one of roughly 25 SMBHs that currently have robust spin measurements determined through X-ray spectroscopy, as described above (see Brenneman 2013 or Reynolds 2014 for a review of these sources). Though theoretical and observational advances in the past decade have revolutionized this field of study, enabling these measurements with accuracy and precision, much work remains to be done in terms of understanding the distribution of SMBH spins in the local universe, as well as any trends between spin and other properties of the AGN or its host galaxy (e.g., accretion rate, black hole mass, host morphology). Ideally, we would like to have a sample of hundreds of SMBH spin measurements from which to determine such correlations, but the ability to increase our sample to such a number is, unfortunately, decades away. The signal-tonoise requirements of the data mandate the need for large collecting areas $\left(>2 \mathrm{~m}^{2}\right)$ over a substantial X-ray bandpass (ideally out to $>200 \mathrm{keV}$ in order to distinguish between the continuum, reflection and absorption with accuracy), and though calorimeters offer the spectral resolution necessary to perform all aspects of this science, improvements in spatial resolution will also ultimately be necessary as we probe to higher redshifts and the need to avoid source confusion becomes dominant. Though the upcoming Athena mission will offer a modest expansion of our sample size and the notional mission concept for the X-ray Surveyor would offer the necessary spatial resolution, SMBH spin science will likely be limited to fewer than 50 sources without an order of magnitude advance in Xray observatory collecting areas. With such an instrument in the future, we could begin to probe the correlations mentioned above, as well as uncover the relative roles of gas accretion and mergers for growing SMBHs over cosmic time (Berti \& Volonteri 2008).

The role of spin in AGN jet production is also a topic that merits much more detailed investigation. While there is a strong theoretical framework linking jet power and black hole spin (Blandford \& Znajek 1977, Garofalo 2009, Tchekhovskoy \& McKinney 2012), observational evidence has been challenging to come by. Due to their shorter duty cycles, microquasars have traditionally made easier targets to use in investigating this phenomenon (Narayan \& McClintock 2012), though significant disagreement persists regarding how the jet power should be measured (Fender, Gallo \& Russell 2010). Spin measurements for radio-loud (RL) AGN are quite rare, however: not only are state changes analogous to those in microquasars seldom seen due to the longer timescales involved (which scale with the mass of the black hole), but the X-ray emission from the jet often complicates the observer's ability to isolate the spectral signatures of relativistic reflection.

3C 120 is the only RLAGN for which a measured spin exists at this time. Overlapping Suzaku and VLBI radio observations by Kataoka et al. (2007) detected dips in the X-ray light curve of the source that were approximately coincident with radio flares, implying that perhaps a disruption of the innermost disk and corona occur when plasma ejection events take place in the jet. Further analysis of Suzaku data by Cowperthwaite \& Reynolds (2012) and Lohfink et al. (2013), the latter of which also employed UV data from Swift, 
has since yielded constraints on both the spin of the SMBH in $3 \mathrm{C} 120(a \geqslant 0.95)$ as well as measurements of the radius of the innermost edge of the disk, demonstrating that the disk has receded in the immediate aftermath of a jet ejection event $\left(r_{\text {in }}=40 \pm 20 r_{\mathrm{g}}\right)$, whereas it extends down to nearly the ISCO in the months leading up to an ejection event $\left(r_{\text {in }}<2 r_{\mathrm{g}}\right)$. The Lohfink et al. (2013) analysis of the Swift/UVOT data also revealed the disk rebuilding after the radio ejection.

Clearly, a larger population of RLAGN with measured SMBH spins is required in order to begin seriously probing the link between spin, jets and AGN/host evolution over cosmic time. In addition to demanding higher effective areas in order to reach fainter targets, this science also underscores the need for a wide X-ray bandpass in order to properly separate the jet spectrum from that of the underlying reflection signatures. The role of accretion disk winds in the overall energy output of these systems is also poorly understood; though there is evidence in microquasars of a balance that is maintained between jet power and wind power (e.g., Neilsen \& Lee 2009), observational evidence of this connection in AGN is harder to come by, again, largely because of the longer duty cycles involved. To further this research, long, coordinated observations are needed that involve both X-ray and radio observatories, at a minimum. Contributions from UV data would also be quite useful in order to track the thermal emission from the disk itself, and to connect the absorption lines seen in outflows from AGN in both X-rays and the UV, which will better enable us to understand their kinematics and ionization structures.

\section{Highly Ionized Gas Atmospheres in AGN}

\section{K. Iwasawa}

The advent of high-resolution gratings spectroscopy of AGN over the past fifteen years has demonstrated that outflows from these systems typically exhibit a range of ionization states, column densities and velocity components (e.g., NGC 5548; Kaastra et al. 2014). The logical expectation is that the most dense, fastest and highly ionized regions of the flow should be found closest to the source of the ionizing photons, i.e., the corona and innermost disk, and indeed, theoretical and observational studies have found that a single outflow in pressure equilibrium can explain the multi-zone absorbing structures that have been used to model many AGN outflows (e.g., NGC 3783, Kaspi et al.2002, Krongold et al. 2003). The ultimate questions this research seeks to answer are, what are the launching radii and physical mechanisms for triggering these outflows, and how much do they contribute to the overall energetics of the AGN/host system?

Investigating the most highly ionized components of these flows gets us closest to their point of origin. These components most prominently manifest as helium-like and hydrogen-like absorption lines of iron (Fexxv and Fexxvi, respectively), seen at restframe energies ranging from $\sim 6.7-7 \mathrm{keV}$. Such ionized lines of iron can also be seen in emission over the same waveband, begging the question of whether we are seeing reemission from the wind itself. Though these lines pale in equivalent width compared to the dominant $\mathrm{Fe} \mathrm{K} \alpha$ emission line at $6.4 \mathrm{keV}$ typically seen in AGN, which can have both distant (torus) and proximal (inner disk) components, they are commonly seen in emission and/or absorption in both type I and type II AGN, including luminous infrared galaxies such as IRAS 00182-7112 (Nandra \& Iwasawa 2007), COSMOS type II AGN at $z>2$ (Iwasawa et al. 2011), and stacked spectra of sub-mm galaxies (Lindner et al. 2012). Evidence for such spectral complexity in the Fe $\mathrm{K}$ band has even been observed in the distant CDF-S source XID403 ( $z=4$; Gilli et al. 2014).

A search for obscured AGN at $z>1.7$ in the XMM-CDFS revealed that hard X-ray excesses are commonly seen when the column density approaches $N_{\mathrm{H}}=10^{24} \mathrm{~cm}^{-2}$ (Comas- 
tri et al.2011, Iwasawa et al. 2012). The advantage of searching for this spectral signature at these redshifts is that rest-frame $10-20 \mathrm{keV}$ enters the $X M M$ bandpass at this point. Using the X-ray color-color diagram based on the rest-frame $3-5 \mathrm{keV}, 5-9 \mathrm{keV}$, and $9-20 \mathrm{keV}$ bands, seven objects were selected for their $9-20 \mathrm{keV}$ excess and were found to be strongly absorbed X-ray sources with column densities of $N_{\mathrm{H}} \geqslant 0.6 \times 10^{24} \mathrm{~cm}^{-2}$, including two possible Compton thick sources. While they are emitting at quasar luminosity, roughly $75 \%$ of the sample objects were found to be absorbed by $N_{\mathrm{H}}>10^{22} \mathrm{~cm}^{-2}$. A comparison with local AGN at the matched luminosity suggests an increasing trend of the absorbed source fraction for high-luminosity AGN towards high redshifts.

Of the seven selected sources, an interesting case is IRAS 18325-5926, a red galaxy with a double radio source containing a Seyfert 2 AGN that is highly variable and moderately absorbed $\left(N_{\mathrm{H}} \sim 3 \times 10^{23} \mathrm{~cm}^{-2}\right)$, with an SMBH mass of $M_{\mathrm{BH}}=2.3 \times 10^{6} \mathrm{M}_{\odot}$. The $X M M-$ determined location of the Fe $\mathrm{K}$ edge yields a redshift of $z=1.59$. In addition to the broad ionized emission line at $6.7 \mathrm{keV}$, the source shows blueshifted absorption features of Fe XXV and Fe XxVI that are variable in flux and show an outflow velocity of $v_{\text {out }}=0.14 c$. This absorption is most prominent during X-ray flares and disappears as the flares decay, implying a transient outflow. Given the velocity and rapid transition speed of this wind, its most likely origin is magnetic, with a launching radius in the inner accretion disk.

\section{The Self-Regulated AGN Feedback Loop: Chaotic Cold Accretion M. Gaspari}

Numerical simulations provide a valuable means with which to investigate the so-called "feedback loop" in AGN without having to rely on observational snapshots of individual galaxies. This latest adaptive mesh refinement (AMR) work zooms in on 3D simulations made with the FLASH4 code, and incorporates the accretion of both cold (molecular) and hot (ionized) gas across physical scales ranging from that of the host galaxy to sub-pc radii, as well as turbulence, cooling, heating and rotation. The types of feedback that these processes generate, which these simulations are designed to investigate, include the total amount of energy released, the deposition of that energy, the mechanical vs. thermal ratio, and local observables such as bubble formation, shocks, metal uplift, and the luminositytemperature relation. Feedback from these processes is then used to alter the initial conditions for the next iteration of the simulation (Gaspari et al.2013, Gaspari 2015).

The ability of these simulations to trace the evolution of turbulence in the intracluster medium (ICM) makes them a valuable tool for investigating SMBH mergers, supernova explosions, galaxy motions and AGN feedback on subsonic scales down to velocities of $\sim 100 \mathrm{~km} \mathrm{~s}^{-1}$ (Gaspari \& Churazov 2013, Gaspari et al.2014). It also enables one to examine the nature and efficiency of both hot and cold accretion under different disk rotation conditions (Gaspari et al.2013, Gaspari 2015) in order to answer the question of whether the SMBH's accretion mode determines the way it provides feedback to the surrounding medium. The assumption is that hot accretion is ordered and rotationsupported, whereas cold accretion follows the Bondi prescription.

When the ICM gas cools efficiently, the combination of turbulence, cooling processes and AGN heating from feedback produces chaotic cold accretion (CCA), rather than accretion that is preferentially directed. In this scenario, cold clouds and filaments lose energy and angular momentum rapidly due to the interaction of these processes. This causes the gas to "rain" down onto SMBH in an episodic fashion that can lead to mass accretion rates of $\dot{M}_{\text {cold }} \sim 100 \dot{M}_{\text {Bondi }}$, roughly equivalent to the cooling rate of the ICM gas. By contrast, when the ICM gas cannot cool efficiently it becomes overheated, and hot accretion results with typical mass accretion rates of $\dot{M}_{\text {hot }} \sim 1 / 3 \dot{M}_{\text {Bondi }}$. The 
temperature profile with radius of the ICM gas in the CCA scenario is roughly flat (Humphrey et al.2009, Wong et al.2014, Russell et al.2015), while the hot accretion scenario results in a cuspy gas temperature profile peaking toward the center of the cluster (Humphrey et al.2008, 2009). Observational evidence for these two types of accretion can be found in SOAR $\mathrm{H} \alpha$ emission spectra from the accreting filaments and clumps (Werner et al.2014), which bear a striking resemblance to the simulations discussed herein (Gaspari 2015).

The CCA scenario would ultimately lead to clumpy, turbulent tori in AGN, which are now being observed (e.g., Tristram et al.2007). Regions of overdensity in the accreted, cold gas could also form cold clouds in broad line region (BLR) and narrow line region (NLR) that can reproduce the variability seen on rapid timescales in so many AGN.

A sketch of how feedback loops operate in AGN and galaxy clusters can be established based in CCA: cooling exceeds heating in the ICM, prompting thermal instabilities to propagate and form cold clumps in the gas. The cold gas then rains down onto the central SMBH, feeding it and boosting its energy output through mechanical feedback in the form of jets, which then heats the ambient medium and suppresses further cooling and gas accretion. Many unanswered questions remain regarding the particulars of this feedback loop, however: the mechanism for converting this accretion into feedback is not yet understood, nor is the timescale for feedback gas "bubbles" to form, expand and cool.

\section{Cosmological, Large-Scale Simulations of Black Hole Growth: Demographics, the AGN-Host Connection and the Relevance of Mergers in Driving Nuclear Activity}

\section{Hirschmann}

Given that observations of AGN show us only snapshots of their lives, large-scale numerical simulations have become a critical tool with which to examine the question of AGN evolution over cosmic time. Recent computational advances have enabled the current generation of simulations to incorporate the most complete array of physical processes yet, including self-consistent radiative cooling (Wiersma, Schaye \& Smith 2009), star formation (Springel \& Hernquist 2003), metal enrichment (Tornatore et al. 2007), black hole growth (via stochastic, Bondi accretion and mergers of non-spinning black holes) and associated feedback processes (Fabian 2012) from both supernovae type II/Ia and AGN. We can therefore use such simulations to investigate the triggering mechanisms of feedback in AGN systems, considering such questions as how the star formation rate of the host is coupled to its AGN's activity and why so many AGN are seen in undisturbed host galaxies across redshift space.

Hirschmann et al. (2014) have performed two such simulations based on the parallel cosmological TreePM-SPH code P-GADGET3 (Springel 2005), one with a large co-moving volume of $500 \mathrm{Mpc}^{3}$ and one with a smaller volume of $68 \mathrm{Mpc}^{3}$ but a factor of almost 20 higher mass resolution. The authors then compared the predicted statistical properties of AGN with results from large observational surveys. Their simulations widely matched the observed black hole properties of the local universe and successfully reproduced the evolution of the bolometric AGN luminosity function at both the low-luminosity and the high-luminosity end up to $z=3.0$ (Hopkins, Gordon \& Hernquist 2007). The lowluminosity end was overestimated by roughly an order of magnitude from $z=1.52 .5$, however, as seen in DeGraf, Matteo \& Springel (2010). The higher resolution run matched the observational data of the low-luminosity end at redshifts $z=34$.

The authors performed a direct comparison of their simulations with the observed soft and hard X-ray luminosity functions of AGN, including an empirical correction for 
torus obscuration, and found similarly good agreement. The observed anti-hierarchical trend in the AGN number density evolution (i.e., the number densities of luminous AGN peak at higher redshifts than those of faint AGN; (Aird et al. 2010)) is self-consistently predicted by these simulations. Overall, this downsizing behavior in the AGN number density as a function of redshift can be mainly attributed to the evolution of the gas density in the resolved vicinity of massive black holes, which is depleted with evolving time as a consequence of star formation and AGN feedback. Observational evidence exists in support of this finding: see (Steinhardt \& Elvis 2010) and (Kelly \& Shen 2013).

An evaluation of the simulated AGN light curves against the timing of their merger episodes reveals a further important correlation: those AGN with $L_{\mathrm{X}}>10^{45} \mathrm{erg} \mathrm{s}^{-1}$ experience growth that is preferentially driven by major mergers, whereas $<20 \%$ of the more moderately luminous AGN show such a trend. Global star formation rates and mass accretion rates peak around $z \sim 1-2$, but it is unclear whether these properties are correlated in individual galaxies; roughly $10 \%$ of AGN are found in starbursting galaxies within the simulations. Both accretion onto the SMBHs and star formation in the host galaxies seem to be triggered by merger events in observational data as well, as inferred from host morphology and galaxy proximity, but it is difficult to establish whether one process causes the other due to selection effects that exist at the luminous end of observed AGN distribution. Further simulations on finer spatial scales (e.g., Hopkins 2015) will be necessary in order to determine the nature of the relationship between accretion, feedback and star formation in AGN hosts.

\section{Probing the Torus Structure of Nearby AGN}

\section{F. Bauer}

X-ray studies of AGN have revealed the near-ubiquitous presence of a neutral, distant reprocessing medium beyond the accretion disk. This so-called molecular torus of Seyfert unification schemes appears to subtend a larger solid angle than the disk, and has been invoked as a means to explain the dichotomy between type I and type II AGN based on how much of this structure crosses our viewing angle to the centralmost regions of the AGN (Antonucci 1993, Urry \& Padovani 1995). Yet beyond it's theorized existence, much about the nature of the torus remains unknown.

We can infer the structure and properties of the torus by examining its reprocessed spectrum of the incident X-ray emission, which manifests primarily in the infrared (e.g., Tristram et al. 2007), and indicates a clumpy structure. We can also analyze the signatures that the torus's gas and dust imprints on AGN X-ray spectra in emission and absorption. These take the form of the narrow fluorescent $\mathrm{Fe} \mathrm{K} \alpha$ line at $6.4 \mathrm{keV}$ and the associated reprocessed continuum emission in the form of the Compton hump, which is shaped by the Fe $\mathrm{K}$ edge on the low-energy end at $7.1 \mathrm{keV}$ and by Compton downscattering at energies above $\sim 20 \mathrm{keV}$ (George \& Fabian 1991). Yaqoob \& Padmanabhan 2004 employed the Chandra/HETG to infer that the narrow $\mathrm{Fe} \mathrm{K} \alpha$ line is present in the vast majority of AGN. Unfortunately, the similar presence of the Compton hump has not yet been as firmly established, owing to a lack of high signal-to-noise data above $10 \mathrm{keV}$. The $N u S T A R$ observatory is currently seeking to change this, however.

The NuSTAR obscured AGN and extragalactic survey programs share a number of scientific goals, chief among which are to resolve the obscured and Compton-thick AGN that are thought to dominate the cosmic X-ray background (CXB). In conjunction with lower-energy, high throughput X-ray observatories such as XMM, Chandra and Suzaku, contemporaneous pointings at such sources as NGC 1068 (Bauer et al. 2015), NGC 4945 (Puccetti et al.2014) and the Circinus galaxy (Arevalo et al.2014) are being obtained 
and analyzed in order to isolate the spectral signatures of the torus. With their focusing abilities and high angular resolution, Chandra and NuSTAR make a particularly powerful pair in terms of separating the cores of nearby AGN from their extended (host galaxy) components both spatially and spectrally, while $X M M$ provides valuable gratings-level spectral resolution at low energies $(<2 \mathrm{keV})$, albeit with reduced angular resolution.

The spectral fits to these sources indicate that the bulk of the Compton hump emission originates from the nucleus (having higher column density), while $>25 \%$ of lower energy reflected flux in these spectra arises from extended emission (with lower column density). This implies that a significant fraction of the Fe K reflection could originate from distances greater than a parsec away from the nucleus (e.g., in NLR clouds), rather than from the sub-pc scales of the torus, as has always been assumed. To further investigate the spatial distribution of reflected emission, then, consider the example of NGC 1068. Bauer et al. (2015) took narrow band images with Chandra at and on either side of the $6.4 \mathrm{keV}$ $\mathrm{Fe} \mathrm{K} \alpha$ line, finding that most of the neutral Fe $\mathrm{K}$ emission comes from extended regions in images taken at $6.4 \mathrm{keV}$, while less extended emission is seen on images taken from either side of that line in energy space. The same trend is seen in both NGC 4945 (Puccetti et al.2014) and Circinus (Arevalo et al.2014), reinforcing the results above and confirming that Fe $\mathrm{K}$ emission from extended structures must be considered when modeling the X-ray spectra of nearby AGN. Given these findings, we can infer that the typical torus structure is most likely clumpy and that reflection occurs via multiple structural components in the AGN system spanning many different spatial scales.

\section{An X-ray Model for Clumpy Tori in AGN}

\section{Y. Liu}

As discussed above, cold absorbing structures are found almost ubiquitously in the X-ray spectra of AGN, with evidence of their presence manifesting in both emission and absorption signatures. Various observations have shown evidence for clumpiness in the absorbing gas, including infrared imaging of the dust structure in the torus itself (Tristram et al. 2007), as well as revolving "clouds" in the BLR (Maiolino et al. 2010). Certain authors have even suggested that the effects of absorption from many layers of clumpy gas can mimic the relativistic inner disk reflection signatures claimed to be observed in many AGN, indicating that SMBH spin measurements made using the reflection method are invalid (Miller, Turner \& Reeves 2008). Though this last argument is disfavored on energetic grounds in most sources (Risaliti et al. 2013, Brenneman 2013, Reynolds 2014), it underscores the need for further theoretical advances in modeling the torus structure in AGN.

Liu \& Li (2014) have constructed an X-ray spectral model for the clumpy torus in AGN by performing simulations using Geant4. Besides the necessary physical processes (e.g., photoelectric absorption, Compton scattering, and fluorescence lines) considered in previous simulations of tori, the authors have included corrections to the treatment of scattering by explicitly considering Rayleigh (coherent) and Compton (incoherent) scattering from bound, rather than free, electrons. This correction can induce a deviation on the X-ray spectra up to $25 \%$ at $1 \mathrm{keV}$, and therefore it cannot be ignored in the simulation of neutral tori.

Different combinations of column density, torus opening angle and number of clouds were investigated by Liu \& Li (2014). The filling factor of the clouds only slightly changes the reflection spectra produced by the torus, while the number of clouds along the line of sight significantly influences the spectra. If there are more clouds, i.e., $\mathrm{N}=10$, the result is similar to the smooth case, while for the extreme case $\left(N_{\mathrm{H}}=10^{25} \mathrm{~cm}^{-2}\right.$ and $\mathrm{N}$ $=2$ ), the shapes of the reflection spectra in different directions are quite similar except 
for the somewhat lower amplitude in the edge-on direction. Therefore, if strong reflection components are found in the observed spectra of type 2 AGN, the "clumpy" scenario could be invoked to explain the spectra.

The anisotropy of the Fe K $\alpha$ line is also impacted by clumpiness in the toroidal medium in a similar way. For $N_{\mathrm{H}}=10^{23} \mathrm{~cm}^{-2}$, the distribution of $\mathrm{Fe} \mathrm{K} \alpha$ photons is nearly isotropic and only slightly changed by the specific parameters of clumpiness, while for $N_{\mathrm{H}}=10^{25} \mathrm{~cm}^{-2}$, a smaller number of clouds significantly degrades the anisotropy of $\mathrm{Fe}$ $\mathrm{K} \alpha$ photons. The strength of the neutral Fe $\mathrm{K} \alpha$ emission line has already been investigated in previous simulations of smooth tori and found to be anisotropic (e.g., Murphy \& Yaqoob 2009). The clumpy torus can further smooth out the anisotropy of this line, depending on the column density of the clouds.

Observationally, the luminosity of the $\mathrm{Fe} \mathrm{K} \alpha$ line will provide independent evidence of the structure of the torus. For example, if there is no significant difference between the luminosities of Fe K $\alpha$ lines in type I and II $\left(N_{\mathrm{H}}>10^{23} \mathrm{~cm}^{-2}\right)$ AGN, the "clumpy" torus is required, barring revision of the unified model. The current observations do not present a conclusive picture. Liu \& Wang (2010) found that the luminosities of the narrow Fe K $\alpha$ lines in Compton-thin and Compton-thick type II AGN are weaker than those in type I AGN by factors of 2.9 and 5.6, respectively. This difference is broadly consistent with the results for a smooth torus. However, from a smaller sample from Chandra/HETG, Shu, Yaqoob \& Wang (2011) found the Fe K $\alpha$ line flux of type I AGN is only marginally lower than that of type II AGN, which requires the smoothing effect of a clumpy torus since the observed column density of Compton-thick AGN is already larger than $N_{\mathrm{H}}=10^{24} \mathrm{~cm}^{-2}$.

In principle, it is possible to combine the infrared spectral energy distribution and Xray spectra to better constrain the structure of tori. However, it should be noted that the gas in the torus is only sensitive to X-ray photons but the dust can also absorb optical photons. Therefore, if the dust-to-gas ratio is not uniform in a torus (which is likely to be the case due to the temperature gradient within this structure), the geometry of the torus probed by the X-ray photons can differ from that obtained from the infrared.

\section{References}

Aird, J. et al. 2010, MNRAS, 401, 2531

Antonucci, R. 1993, ARAA, 31, 473

Arevalo, P. et al. 2014, ApJ, 791, 81

Bauer, F. E. et al. 2015, ApJ, 812, 116

Berti, E. \& Volonteri, M. 2008, ApJ, 684, 822

Blandford, R. D. \& Znajek, R. L. 1977, MNRAS, 179, 433

Bower, R. G. et al. 2006, MNRAS, 370, 645

Brenneman, L. W. \& Reynolds, C. S. 2006, ApJ, 652, 1028

Brenneman, L. 2013, arXiv:1309.6334

Brenneman, L. W. et al. 2011, ApJ, 736, 103

Chartas, G. et al. 2012, ApJ, 757, 137

Comastri, A. et al. 2011, A\&GA, 526L, 9

Cowperthwaite, P. \& Reynolds, C. S. 2012, ApJ, 752L, 21

Dauser, T. et al. 2013, MNRAS, 430, 1694

Dauser, T. et al. 2010, MNRAS, 409, 1534

DeGraf, C., Di Matteo, T. \& Springel, V. 2010, MNRAS, 402, 1927

Fabian, A. C. 2012, ARAA, 50, 455

Fabian, A. C. et al. 1989, MNRAS, 238, 729

Fender, R. P., Gallo, E., \& Russell, D. 2010, MNRAS, 406, 1425

Garcia, J. et al. 2014, ApJ, 782, 76 
Garcia, J. et al. 2013, ApJ, 768146

Garofalo, D. 2009, ApJ, 699, 400

Gaspari, M. 2015, MNRAS, 451L, 60

Gaspari, M. et al. 2014, ApJ, 783L, 10

Gaspari, M. et al. 2013, MNRAS, 432, 3401

Gaspari, M. \& Churazov, E. 2013, A\& A, 559A, 78

George, I. M. \& Fabian, A. C. 1991, MNRAS, 249, 352

Gilli, R. et al. 2014, A\&A A, 562, 67

Halpern, J. P. 1984, ApJ, 281, 90

Harrison, F. A. et al. 2013, ApJ, 770103

Hirschmann, M. et al. 2014, MNRAS, 442, 2304

Hopkins, P. F. 2015, MNRAS, 450, 53

Hopkins, P. F., Gordon, T. \& Hernquist, L. 2007, ApJ, 654, 731

Humphrey, P. J. et al. 2009, ApJ, 703, 1257

Humphrey, P. J. et al. 2008, ApJ, 683, 161

Iwasawa, K. et al. 2012, $A \mathscr{E} A, 546 \mathrm{~A}, 84$

Iwasawa, K. et al. 2011, $A \& A$ A, 529A, 106

Kaastra, J. S. et al. 2014, Science, 345, 64

Kaspi, S. et al. 2002, ApJ, 574, 643

Kataoka, J. et al. 2007, PASJ, 59, 279

Kelly, B. C. \& Shen, Y. 2013, ApJ, 764, 45

Krongold, Y. et al. 2003, ApJ, 597, 832

Laor, A. 1991, ApJ, 376, 90

Lindner, R. R. et al. 2012, ApJ, 757, 3

Liu, Y. \& Li, X. 2014, ApJ, 787, 52

Liu, T. \& Wang, J.-X. 2010, ApJ, 725, 2381

Lohfink, A. et al. 2013, ApJ, 772, 83L

Maiolino, R. et al. 2010, A\&̊A, 517A, 47

McClintock, J. E., Narayan, R., \& Steiner, J. F. 2014, Space Sci. Revs., 183, 295

McConnell, N. J. \& Ma, C.-P. 2013, ApJ, 764, 184

Miller, L., Turner, T. J., \& Reeves, J. N. 2008, $A \mathscr{E} A$ A, 483, 437

Mosquera, A. M. et al. 2013, ApJ, 769, 53

Murphy, K. D. \& Yaqoob, T. 1009, MNRAS, 397, 1549

Nandra, K. et al. 2013, arXiv:1306.2307

Nandra, K. \& Iwasawa, K. 2007, MNRAS, 382L, 1

Narayan, R. \& McClintock, J. E. 2012, MNRAS, 419L, 69

Neilsen, J. \& Lee, J. C. 2009, Nature, 458, 481

Puccetti, S. et al. 2014, ApJ, 793, 26

Reynolds, C. S. 2014, Space Sci. Revs., 183, 277

Reynolds, C. S. 1997, MNRAS, 286, 513

Reynolds, C. S. \& Fabian, A. C. 2008, ApJ, 675, 1048

Reynolds, C. S. \& Nowak, M. A. 2003, Phys. Reps., 377, 389

Risaliti, G. et al. 2013, Nature, 494, 449

Ross, R. R. \& Fabian, A. C. 2005, MNRAS, 358, 211

Russell, H. R. et al. 2015, MNRAS, 451, 588

Shu, X. W., Yaqoob, T., \& Wang, J. X. 2011, ApJ, 738, 147

Springel, V. 2005, MNRAS, 364, 1105

Springel, V. \& Hernquist, L. 2003, MNRAS, 339, 289

Steinhardt, C. L. \& Elvis, M. 2010, MNRAS, 402, 2637

Tchekhovskoy, A. \& McKinney, J. P. 2012, MNRAS, 423L, 55

Tornatore, L. et al. 2007, MNRAS, 382, 1050

Tristram, K. R. W. et al. 2007, AN, 328, 673

Urry, C. M. \& Padovani, P. 1995, PASP, 107, 803

Walton, D. J. et al. 2014, ApJ, 788, 76 
Weisskopf, M. C. et al. 2015, SPIE, 9510E, 2

Werner, N. et al. 2014, MNRAS, 439, 2291

Wiersma, R. P. C., Schaye, J. \& Smith, B. D. 2009, MNRAS, 393, 99

Wong, K.-W. et al. 2014, ApJ, 780, 9

Wu, X.-B. et al. 2015, Nature, 518, 512

Yaqoob, T. \& Padmanabhan, U. 2004, ApJ, 604, 63 